\title{
Another Newton-type method with $(k+2)$ order convergence for solving quadratic equations
}

\author{
R. Thukral \\ Padé Research Centre, 39 Deanswood Hill, Leeds, West Yorkshire, LS17 5JS, England
}

\begin{abstract}
In this paper we define another Newton-type method for finding simple root of quadratic equations. It is proved that the new one-point method has the convergence order of $(k+2)$ requiring only three function evaluations per full iteration, where $k$ is the number of terms in the generating series. The Kung and Traub conjecture states that the multipoint iteration methods, without memory based on $n$ function evaluations, could achieve maximum convergence order $2^{n-1}$ but, the new method produces convergence order of nine, which is better than the expected maximum convergence order. Finally, we have demonstrated that our present method is very competitive with the similar methods.
\end{abstract}

Keywords: Newton method; Quadratic equations; Kung-Traub's conjecture; Efficiency index; Optimal order of convergence.

Subject Classifications: AMS (MOS): 65H05.

\section{INTRODUCTION}

In this paper, we present a new one-point $(k+2)$-order iterative method to find a simple root of the nonlinear equation. Many higher order multi-point variants of the Newton method have been developed based on the Kung and Traub conjecture [4]. Here we present a new iterative method which has a better efficiency index than the classical Newton method [3,5,6,9] and is equivalent to the Thukral [8], the Ahmad [1] and Babajee [2] method. We have found that the main difference between the proposed method and the methods given in [1,2] is that the proposed method is based on onepoint and is much simpler to construct. For the purpose of this paper, we improve the classical Newton method and to construct a new $(k+2)$-order iterative method for finding simple roots of quadratic equations. The new method presented in this paper only uses three evaluations of the function per iteration. Kung and Traub conjectured that the multipoint iteration methods, without memory based on $n$ evaluations, could achieve optimal convergence order $2^{n-1}$. In fact, we have obtained a higher order of convergence than the maximum order of convergence suggested by Kung and Traub conjecture [4]. We demonstrate that the Kung and Traub conjecture fails for a particular case.

\section{PRELIMINARIES}

Here we state the essential definitions applicable to an iterative method.

Definition 1 Let $f(x)$ be a real function with a simple root $\alpha$ and let $\left\{x_{n}\right\}$ be a sequence of real numbers that converge towards $\alpha$. The order of convergence $p$ is given by

$\lim _{n \rightarrow \infty} \frac{x_{n+1}-\alpha}{\left(x_{n}-\alpha\right)^{p}}=\lambda \neq 0$

where $p \in \mathfrak{R}^{+}$and $\lambda$ is the asymptotic error constant. Let $e_{n}=x_{n}-\alpha$ be the error in the $n$th iteration, then the relation

$e_{n+1}=\zeta e_{n}^{p}+\mathrm{O}\left(e_{n}^{p+1}\right)$

is the error equation. If the error equation exists, then $p$ is the order of convergence of the iterative method, $[3,5,6,9]$.

Definition 2 Let $n$ be the number of function evaluations of the iterative method. The efficiency of the iterative method is measured by the concept of efficiency index and defined as

$$
E(n, p)=\sqrt[n]{p}
$$

where $p$ is the order of convergence of the method, [6]. 
Definition 3 (Kung and Traub conjecture) Let $x_{n+1}=g\left(x_{n}\right)$ define as an iterative function without memory with $n$ evaluations. Then

$$
p(g) \leq p_{\text {opt }}=2^{n-1}
$$

where $p_{\text {opt }}$ is the maximum order, [4].

Definition 4 Suppose that $x_{n-1}, x_{n}$ and $x_{n+1}$ are three successive iterations closer to the root $\alpha$. Then the computational order of convergence may be approximated by the following;

$$
\hat{p}_{1} \approx \frac{\ln \left|\delta_{n} \delta_{n-1}^{-1}\right|}{\ln \left|\delta_{n-1} \delta_{n-2}^{-1}\right|},
$$

$\hat{p}_{2} \approx \frac{\ln \left|f\left(x_{n}\right) f\left(x_{n-1}\right)^{-1}\right|}{\ln \left|f\left(x_{n-1}\right) f\left(x_{n-2}\right)^{-1}\right|}$,

$\hat{p}_{3} \approx \frac{\ln \left|\left(\Delta x_{n}\right)\left(\Delta x_{n-1}\right)^{-1}\right|}{\ln \left|\left(\Delta x_{n-1}\right)\left(\Delta x_{n-2}\right)^{-1}\right|}$,

$$
\hat{p}_{4} \approx \frac{\ln \left|\left(x_{n}-\alpha\right)\left(x_{n-1}-\alpha\right)^{-1}\right|}{\ln \left|\left(x_{n-1}-\alpha\right)\left(x_{n-2}-\alpha\right)^{-1}\right|},
$$

where $\delta_{i}=f\left(x_{i}\right) f^{\prime}\left(x_{i}\right)^{-1}, \Delta x_{i}=x_{i+1}-x_{i}$. The formulas (5)-(8) are given in [5,7-9]. Let $k$ be the total number of function evaluations per iteration.

\section{NEW ONE-POINT METHOD AND ANALYSIS OF CONVERGENCE}

In this section we define a new class of one-point $(k+2)$-order method for finding simple roots of a quadratic equation. In fact, the new Newton-type iterative method is an improvement of the classical Newton method. We shall demonstrate that the new two-point method can be constructed to produce any desired order of convergence and is equivalent to the Thukral [8], the Ahmad [1] and Babajee [2] two-point methods. The order of convergence the new iterative method is determined by the $k$, number of terms in the generating series, which improves the classical Newton method. First, we will state the essentials of the Thukral, Ahmad and Babajee methods, hence we will illustrate the equivalency of the new onepoint method.

\section{The Thukral two-point Method [8]}

The two-point $(k+2)$-order Newton-type method is expressed by

$$
\begin{aligned}
& y_{n}=x_{n}-u\left(x_{n}\right) \\
& x_{n+1}=x_{n}-u\left(x_{n}\right) G(z, k)
\end{aligned}
$$

where

$$
\begin{aligned}
& G(z, k)=1+\sum_{i=1}^{k} a_{i} z^{i} \\
& u\left(x_{n}\right)=\frac{f\left(x_{n}\right)}{f^{\prime}\left(x_{n}\right)} \text { and } z=\frac{f\left(y_{n}\right)}{f\left(x_{n}\right)},
\end{aligned}
$$

The first eight constants coefficients of the terms of (11) are 
$a_{1}=1, \quad a_{2}=2, \quad a_{3}=5, \quad a_{4}=14, \quad a_{5}=42, \quad a_{6}=132, \quad a_{7}=429, \quad a_{8}=1430$

where $x_{0}$ is the initial guess and provided that denominators of (12) are not equal to zero. Now, we shall verify the convergence property of the new two-point $(k+2)$-order iterative method (10).

\section{The Ahmad and Babajee two-point Method [1,2]}

Ahmad and Babajee presented a two-point $(k+2)$-order method which is expressed by

$$
\begin{aligned}
& y_{n}=x_{n}-\left(\frac{2}{3}\right) u\left(x_{n}\right) \\
& x_{n+1}=x_{n}-u(x) H(\tau, k)
\end{aligned}
$$

where

$$
H(\tau, k)=1+\sum_{i=0}^{k-1} a_{i}(\tau-1)^{i}, u(x)=\frac{f\left(x_{n}\right)}{f^{\prime}\left(x_{n}\right)} \text { and } \tau=\frac{f^{\prime}\left(y_{n}\right)}{f^{\prime}\left(x_{n}\right)},
$$

The first six constants coefficients of the terms of (16) are

$a_{0}=\frac{3}{4}, \quad a_{1}=\frac{9}{8}, \quad a_{2}=\frac{135}{64}, \quad a_{3}=\frac{567}{128}, \quad a_{4}=\frac{5103}{512}, \quad a_{5}=\frac{24057}{1024}$,

and the coefficients $a_{i}$ is calculated by

$a_{k+1}=C_{k}\left(-\frac{3}{4}\right)^{k+1}$

where $C_{k}$ is the asymptotic error constant, obtained by the previous order of the iterative method [2].

\section{The new one-point Method}

The one-point $(k+2)$-order Newton-type method is expressed by

$x_{n+1}=x_{n}-u\left(x_{n}\right) J\left(u\left(x_{n}\right), v\left(x_{n}\right) ; k\right)$

where

$$
\begin{aligned}
& J\left(u\left(x_{n}\right), v\left(x_{n}\right) ; k\right)=1+\sum_{i=1}^{k} a_{i}\left(u\left(x_{n}\right) v\left(x_{n}\right)\right)^{i} \\
& u\left(x_{n}\right)=\frac{f\left(x_{n}\right)}{f^{\prime}\left(x_{n}\right)} \text { and } v\left(x_{n}\right)=2^{-1} \frac{f^{\prime \prime}\left(x_{n}\right)}{f^{\prime}\left(x_{n}\right)},
\end{aligned}
$$

The first eight constants coefficients of the terms of (20) are

$$
a_{1}=1, \quad a_{2}=2, \quad a_{3}=5, \quad a_{4}=14, \quad a_{5}=42, \quad a_{6}=132, \quad a_{7}=429, \quad a_{8}=1430
$$

where $x_{0}$ is the initial guess and provided that denominators of (21) are not equal to zero. Now, we shall verify the convergence property of the new one-point $(k+2)$-order iterative method (19).

Theorem 1 : Let $\alpha \in D$ be a simple zero of a sufficiently smooth function $f: D \subset \Re \rightarrow \Re$ for an open interval $D$. If the initial guess $x_{0}$ is sufficiently close to $\alpha$, then the convergence order of the new one-point iterative method defined by $(19)$ is $(k+2)$. 


\section{Proof}

Let $\alpha$ be a simple root of $f(x)$, i.e. $f(\alpha)=0$ and $f^{\prime}(\alpha) \neq 0$, and the error is expressed as $e=x-\alpha$.

The Taylor series expansion and taking into account $f(\alpha)=0$, we have

$f\left(x_{n}\right)=f^{\prime}(\alpha)\left(e_{n}+c_{2} e_{n}^{2}\right)$.

$f^{\prime}\left(x_{n}\right)=f^{\prime}(\alpha)\left(1+2 c_{2} e_{n}\right)$.

$f^{\prime \prime}\left(x_{n}\right)=f^{\prime}(\alpha)\left(2 c_{2}\right)$.

where

$c_{2}=\frac{f^{\prime \prime}(\alpha)}{f^{\prime}(\alpha)}$.

Dividing (23) by (24), we have

$\frac{f\left(x_{n}\right)}{f^{\prime}\left(x_{n}\right)}=e_{n}-c_{2} e_{n}^{2}+2 c_{2}^{2} e_{n}^{3}-4 c_{2}^{3} e_{n}^{4}+8 c_{2}^{3} e_{n}^{4} \cdots$

and

$\left(\frac{f^{\prime \prime}\left(x_{n}\right)}{f^{\prime}\left(x_{n}\right)}\right)=c_{2} e_{n}-3 c_{2}^{2} e_{n}^{2}+8 c_{2}^{3} e_{n}^{3}-20 c_{2}^{4} e_{n}^{4}+48 c_{2}^{5} e_{n}^{5}-\cdots$.

Substituting (27) in (9), we obtain

$e_{n+1}=e_{n}-\frac{f\left(x_{n}\right)}{f^{\prime}\left(x_{n}\right)}$,

$\operatorname{AEC}(1)=c_{2} e_{n}^{2}$.

It is well known that (30) is the asymptotic error constant for the classical Newton method. Therefore, we take the coefficient of the error equation (30) as our first coefficient of the generating series given in (20) as $a_{1}=1$. Furthermore, we obtain a family of higher iterative method by increasing the terms of summation series of (20). Hence, we show the asymptotic error constant $A E C(k+1)$ for the $(k+2)$-order Newton-type method. The next seven members of (19) with their error equation are

1. $k=1$ : One-point third-order iterative method is given by

$x_{n+1}=x_{n}-u\left(x_{n}\right)\left[1+u\left(x_{n}\right) v\left(x_{n}\right)\right]$

and the error equation

$A E C(2)=2 c_{2}^{2} e_{n}^{3}$.

2. $k=2$ : One-point fourth-order iterative method is given by

$x_{n+1}=x_{n}-u\left(x_{n}\right)\left[1+u\left(x_{n}\right) v\left(x_{n}\right)+2\left(u\left(x_{n}\right) v\left(x_{n}\right)\right)^{2}\right]$

and the error equation

$\operatorname{AEC}(3)=5 c_{2}^{3} e_{n}^{4}$.

3. $k=3$ : One-point fifth-order iterative method is given by 
$x_{n+1}=x_{n}-u\left(x_{n}\right)\left[1+u\left(x_{n}\right) v\left(x_{n}\right)+2\left(u\left(x_{n}\right) v\left(x_{n}\right)\right)^{2}+5\left(u\left(x_{n}\right) v\left(x_{n}\right)\right)^{3}\right]$

and the error equation

$A E C(4)=14 c_{2}^{4} e_{n}^{5}$.

4. $k=4$ : One-point sixth-order iterative method is given by

$x_{n+1}=x_{n}-u\left(x_{n}\right)\left[1+u\left(x_{n}\right) v\left(x_{n}\right)+2\left(u\left(x_{n}\right) v\left(x_{n}\right)\right)^{2}+5\left(u\left(x_{n}\right) v\left(x_{n}\right)\right)^{3}+14\left(u\left(x_{n}\right) v\left(x_{n}\right)\right)^{4}\right]$

and the error equation

$$
\operatorname{AEC}(5)=42 c_{2}^{5} e_{n}^{6} \text {. }
$$

5. $k=5$ : One-point seventh-order iterative method is given by

$$
\begin{aligned}
x_{n+1}=x_{n}- & u\left(x_{n}\right)\left[1+u\left(x_{n}\right) v\left(x_{n}\right)+2\left(u\left(x_{n}\right) v\left(x_{n}\right)\right)^{2}+5\left(u\left(x_{n}\right) v\left(x_{n}\right)\right)^{3}\right. \\
+ & \left.14\left(u\left(x_{n}\right) v\left(x_{n}\right)\right)^{4}+42\left(u\left(x_{n}\right) v\left(x_{n}\right)\right)^{5}\right]
\end{aligned}
$$

and the error equation

$$
\operatorname{AEC}(6)=132 c_{2}^{6} e_{n}^{7} \text {. }
$$

6. $k=6$ : One-point eighth-order iterative method is given by

$$
\begin{aligned}
x_{n+1}=x_{n}- & u\left(x_{n}\right)\left[1+u\left(x_{n}\right) v\left(x_{n}\right)+2\left(u\left(x_{n}\right) v\left(x_{n}\right)\right)^{2}+5\left(u\left(x_{n}\right) v\left(x_{n}\right)\right)^{3}\right. \\
+ & \left.14\left(u\left(x_{n}\right) v\left(x_{n}\right)\right)^{4}+42\left(u\left(x_{n}\right) v\left(x_{n}\right)\right)^{5}+132\left(u\left(x_{n}\right) v\left(x_{n}\right)\right)^{6}\right]
\end{aligned}
$$

and the error equation

$$
\operatorname{AEC}(7)=429 c_{2}^{7} e_{n}^{8} \text {. }
$$

It is well established that the maximum order of convergence of optimal methods with three functions evaluations is 4 . As illustrated above we have obtained order of convergence greater than 4 , hence the Kung and Traub conjecture fails for this particular case. To produce the next one-point higher order of convergence with only three function evaluations, we use the previous coefficient of the error equation as our coefficient $a_{k}$ of the next term of the generating series, thus we can calculate the next higher order of convergence method.

\section{NUMERICAL EXAMPLES}

The present one-point $(k+2)$-order method given by $(11)$ is employed to solve quadratic equations with a simple root. To demonstrate the performance of the new one-point $(k+2)$-order method, three particular quadratic equations are used. The difference between the simple root $\alpha$ and the approximation $x_{n}$ for test functions with initial guess $x_{0}$ are displayed in tables. In fact, $x_{n}$ is calculated by using the same total number of function evaluations for all methods. Furthermore, the computational order of convergence approximations (COC) are displayed in tables. The numerical computations listed in the tables were performed on an algebraic system called Maple. In fact, the errors displayed are of absolute value.

\subsection{Numerical example 1}

In our first example we will demonstrate the convergence of the new one-point Newton-type method for the following quadratic equation

$$
f(x)=x^{2}-5 x+1
$$


and the exact value of the simple root of (43) is $\alpha=0.208712153 \ldots$ In Table 1 the errors obtained by the Newton-type method described, are based on number of terms used in (20) and taking the initial value $x_{0}=-2^{-1}$.

Table 1 Errors occurring in the estimates of the root of (43)

\begin{tabular}{cccccc}
\hline Method (19) & $\left|x_{1}-\alpha\right|$ & $\left|x_{2}-\alpha\right|$ & $\left|x_{3}-\alpha\right|$ & $\left|x_{4}-\alpha\right|$ & $\left|f\left(x_{4}\right)\right|$ \\
$k=0$ & $0.212 \mathrm{e}-1$ & $0.973 \mathrm{e}-4$ & $0.207 \mathrm{e}-8$ & $0.931 \mathrm{e}-18$ & $0.427 \mathrm{e}-17$ \\
$k=1$ & $0.320 \mathrm{e}-2$ & $0.314 \mathrm{e}-8$ & $0.294 \mathrm{e}-26$ & $0.241 \mathrm{e}-80$ & $0.111 \mathrm{e}-79$ \\
$k=2$ & $0.613 \mathrm{e}-3$ & $0.732 \mathrm{e}-14$ & $0.149 \mathrm{e}-57$ & $0.257 \mathrm{e}-232$ & $0.118 \mathrm{e}-231$ \\
$k=3$ & $0.132 \mathrm{e}-3$ & $0.129 \mathrm{e}-20$ & $0.112 \mathrm{e}-105$ & $0.548 \mathrm{e}-531$ & $0.251 \mathrm{e}-530$ \\
$k=4$ & $0.307 \mathrm{e}-4$ & $0.174 \mathrm{e}-28$ & $0.584 \mathrm{e}-174$ & $0.821 \mathrm{e}-1047$ & 0.3761046 \\
$k=5$ & $0.749 \mathrm{e}-5$ & $0.188 \mathrm{e}-37$ & $0.119 \mathrm{e}-265$ & $0.482 \mathrm{e}-1863$ & $0.221 \mathrm{e}-1862$ \\
$k=6$ & $0.189 \mathrm{e}-5$ & $0.165 \mathrm{e}-47$ & $0.564 \mathrm{e}-384$ & $0.104 \mathrm{e}-3075$ & $0.475 \mathrm{e}-3075$ \\
\hline
\end{tabular}

Table 2 Computational order of convergence $\hat{p}_{i}$

\begin{tabular}{cccccc}
\hline Method (19) & $\hat{p}_{1}$ & $\hat{p}_{2}$ & $\hat{p}_{3}$ & $\hat{p}_{4}$ & $p_{\text {opt }}$ \\
$k=0$ & 2.0000 & 2.0000 & 0.8505 & 2.0000 & 2 \\
$k=1$ & 3.0000 & 3.0002 & 2.9474 & 3.0001 & 4 \\
$k=2$ & 4.0000 & 4.0000 & 3.8553 & 4.0000 & 4 \\
$k=3$ & 5.0000 & 5.0000 & 4.9679 & 5.0000 & 4 \\
$k=4$ & 6.0000 & 6.0000 & 5.9458 & 6.0000 & 4 \\
$k=5$ & 7.0000 & 7.0000 & 6.9761 & 7.0000 & 4 \\
$k=6$ & 8.0000 & 8.0000 & 7.9678 & 8.0000 & 4 \\
\hline
\end{tabular}

\subsection{Numerical example 2}

In our second example we will demonstrate the convergence of new Newton-type iterative method for a different type of quadratic equation

$$
f(x)=x^{2}+8 x+2 \text {, }
$$

and the exact value of the simple root of (44) is $\alpha=-7.74165739 \ldots$. In Table 1 the errors obtained by the Newtontype method described, are based on number of terms used in (20) and taking the initial value $x_{0}=-8$. 
Table 3 Errors occurring in the estimates of the root of (44)

\begin{tabular}{cccccc}
\hline Method (19) & $\left|x_{1}-\alpha\right|$ & $\left|x_{2}-\alpha\right|$ & $\left|x_{3}-\alpha\right|$ & $\left|x_{4}-\alpha\right|$ & $\left|f\left(x_{4}\right)\right|$ \\
$k=0$ & $0.834 \mathrm{e}-2$ & $0.928 \mathrm{e}-5$ & $0.115 \mathrm{e}-10$ & $0.177 \mathrm{e}-22$ & $0.132 \mathrm{e}-21$ \\
$k=1$ & $0.530 \mathrm{e}-3$ & $0.532 \mathrm{e}-11$ & $0.537 \mathrm{e}-35$ & $0.554 \mathrm{e}-107$ & $0.415 \mathrm{e}-106$ \\
$k=2$ & $0.418 \mathrm{e}-4$ & $0.365 \mathrm{e}-19$ & $0.213 \mathrm{e}-79$ & $0.244 \mathrm{e}-320$ & $0.182 \mathrm{e}-319$ \\
$k=3$ & $0.369 \mathrm{e}-5$ & $0.303 \mathrm{e}-29$ & $0.115 \mathrm{e}-149$ & $0.885 \mathrm{e}-752$ & $0.662 \mathrm{e}-751$ \\
$k=4$ & $0.347 \mathrm{e}-6$ & $0.313 \mathrm{e}-41$ & $0.169 \mathrm{e}-251$ & $0.416 \mathrm{e}-1513$ & $0.312 \mathrm{e}-1512$ \\
$k=5$ & $0.342 \mathrm{e}-7$ & $0.413 \mathrm{e}-55$ & $0.154 \mathrm{e}-390$ & $0.153 \mathrm{e}-2738$ & $0.115 \mathrm{e}-2737$ \\
$k=6$ & $0.349 \mathrm{e}-8$ & $0.711 \mathrm{e}-71$ & $0.212 \mathrm{e}-572$ & $0.136 \mathrm{e}-4584$ & $0.101 \mathrm{e}-4583$ \\
\hline
\end{tabular}

Table 4 Computational order of convergence $\hat{p}_{i}$

\begin{tabular}{cccccc}
\hline Method (19) & $\hat{p}_{1}$ & $\hat{p}_{2}$ & $\hat{p}_{3}$ & $\hat{p}_{4}$ & $p_{\text {opt }}$ \\
$k=0$ & 2.0000 & 2.0000 & 2.0374 & 2.0000 & 2 \\
$k=1$ & 3.0000 & 3.0000 & 3.0205 & 3.0000 & 4 \\
$k=2$ & 4.0000 & 4.0000 & 4.0145 & 4.0000 & 4 \\
$k=3$ & 5.0000 & 5.0000 & 5.0113 & 5.0000 & 4 \\
$k=4$ & 6.0000 & 6.0000 & 6.0094 & 6.0000 & 4 \\
$k=5$ & 7.0000 & 7.0000 & 7.0081 & 7.0000 & 4 \\
$k=6$ & 8.0000 & 8.0000 & 8.0071 & 8.0000 & 4 \\
\hline
\end{tabular}

\subsection{Numerical example 3}

In this subsection we take another cubic equation. We will demonstrate the convergence of the new Newton-type iterative method for the following quadratic equation

$$
f(x)=x^{2}-3 x-9 \text {, }
$$

and the exact value of the simple root of (45) is $\alpha=-1.85410197 \ldots$. In Table 1 the errors obtained by the Newtontype method described, are based on number of terms used in (20) and taking the initial value $x_{0}=-2$.

Table 5 Errors occurring in the estimates of the root of (45)

\begin{tabular}{cccccc}
\hline Method (19) & $\left|x_{1}-\alpha\right|$ & $\left|x_{2}-\alpha\right|$ & $\left|x_{3}-\alpha\right|$ & $\left|x_{4}-\alpha\right|$ & $\left|f\left(x_{4}\right)\right|$ \\
$k=0$ & $0.304 \mathrm{e}-2$ & $0.138 \mathrm{e}-5$ & $0.283 \mathrm{e}-12$ & $0.119 \mathrm{e}-25$ & $0.799 \mathrm{e}-25$ \\
$k=1$ & $0.125 \mathrm{e}-3$ & $0.877 \mathrm{e}-13$ & $0.300 \mathrm{e}-40$ & $0.120 \mathrm{e}-122$ & $0.805 \mathrm{e}-122$ \\
$k=2$ & $0.644 \mathrm{e}-5$ & $0.285 \mathrm{e}-22$ & $0.109 \mathrm{e}-91$ & $0.237 \mathrm{e}-369$ & $0.159 \mathrm{e}-368$ \\
$k=3$ & $0.370 \mathrm{e}-6$ & $0.477 \mathrm{e}-34$ & $0.171 \mathrm{e}-173$ & $0.101 \mathrm{e}-870$ & $0.674 \mathrm{e}-870$ \\
$k=4$ & $0.227 \mathrm{e}-7$ & $0.423 \mathrm{e}-48$ & $0.177 \mathrm{e}-292$ & $0.958 \mathrm{e}-1759$ & $0.643 \mathrm{e}-1758$ \\
$k=5$ & $0.146 \mathrm{e}-8$ & $0.204 \mathrm{e}-64$ & $0.214 \mathrm{e}-455$ & $0.302 \mathrm{e}-3192$ & $0.203 \mathrm{e}-3191$ \\
$k=6$ & $0.970 \mathrm{e}-10$ & $0.549 \mathrm{e}-83$ & $0.576 \mathrm{e}-669$ & $0.847 \mathrm{e}-5357$ & $0.568 \mathrm{e}-5356$
\end{tabular}


Table 6 Computational order of convergence $\hat{p}_{i}$

\begin{tabular}{cccccc}
\hline Method (19) & $\hat{p}_{1}$ & $\hat{p}_{2}$ & $\hat{p}_{3}$ & $\hat{p}_{4}$ & $p_{\text {opt }}$ \\
$k=0$ & 2.0000 & 2.0000 & 2.0214 & 2.0000 & 2 \\
$k=1$ & 3.0000 & 3.0000 & 3.0114 & 3.0000 & 4 \\
$k=2$ & 4.0000 & 4.0000 & 4.0080 & 4.0000 & 4 \\
$k=3$ & 5.0000 & 5.0000 & 5.0062 & 5.0000 & 4 \\
$k=4$ & 6.0000 & 6.0000 & 6.0051 & 6.0000 & 4 \\
$k=5$ & 7.0000 & 7.0000 & 7.0044 & 7.0000 & 4 \\
$k=6$ & 8.0000 & 8.0000 & 8.0039 & 8.0000 & 4 \\
\hline
\end{tabular}

\section{REMARKS AND CONCLUSION}

In this study, a new one-point $(k+2)$-order Newton-type method has been presented. The prime motive for presenting the new class of iterative method was to improve the classical Newton method. The new one-point iterative method requires three function evaluations and has the order of convergence $(k+2)$. The efficiency index of the new method is determined by definition 2. Hence, the efficiency index of the new iterative method given by (19) is $\sqrt[3]{k+2}$ and the efficiency index of the classical Newton method is $\sqrt[2]{2}$. The main difference between the new method and the established method $[1,2,8]$ is that the new method is based on one-point iterative and formation of the generating series is completely different. We have found that the error equations of the new method and the Thukral, Ahmad and Babajee methods are identical. Furthermore, the coefficients of the generating series in the new method are obtained naturally, whereas the coefficients of the generating series in the Ahmad and Babajee method requires much more calculations. Therefore, it is evident that the coefficient in the generating series of the new method is much simpler than the Thukral, Ahmad and Babajee' method. The essential advantages of the new method are: very high computational efficiency; the new method is not limited to the Kung and Traub conjecture; better efficiency index than the classical Newton method; simple one-step iteration method. Finally, we conjecture that the proposed method may extended to higher order polynomials.

\section{REFERENCES}

[1] F. Ahmad, Higher order iterative methods for solving matrix vector equations (2015), Researchgate, doi: 10.13140/RG.2.1.1519.2487.

[2] D. K. R. Babajee, On the Kung-Traub conjecture for iterative methods for solving quadratic equations, Algorithms 2016, 9, 1, doi 10.3390/a9010001.

[3] W. Gautschi, Numerical Analysis: an Introduction, Birkhauser, 1997.

[4] H. Kung, J. F. Traub, Optimal order of two-point and multipoint iteration, J. Assoc. Comput. Math. 21 (1974) 643651.

[5] M. S. Petkovic, B. Neta, L. D. Petkovic, J. Dzunic, Multipoint methods for solving nonlinear equations, Elsevier 2012.

[6] A. M. Ostrowski, Solutions of equations and system of equations, Academic Press, New York, 1960.

[7] R. Thukral, New modifications of Newton-type methods with eighth-order convergence for solving nonlinear equations, J. Adv. Math. Vol 10 (3) (2015) 3362-3373.

[8] R. Thukral, New improved Newton method with $(k+2)$-order convergence for solving quadratic equations, Euro. J. math. Computer. Sci. 3 (1) (2016).

[9] J. F. Traub, Iterative Methods for solution of equations, Chelsea publishing company, New York 1977. 